\title{
REVISIÓN DE LA TÉCNICA “EL JUEGO DEL BUEN COM- PORTAMIENTO"
}

\author{
Ma del Rosario Rulz Olivares \\ Ma José Pino Osuna \\ Javier Herruzo Cabrera
}

Área de Personalidad, Evaluación y Tratamiento Psicológico. Departamento de Psicologla. Universidad de Córdoba

\section{RESUMEN}

El Juego del Buen Comportamiento, JBC (Good behavior Game, GBG), tiene como objetivo fundamental reducir la frecuencia de determinados comportamientos disruptivos en el aula como gritar, pelearse, interrumpir, etc. Dichos comportamientos se caracterizan por ser funcionales en ciertos contextos como el recreo o el juego libre entre compañeros, $y$ disfuncionales en situaciones educativas. El objetivo de este artículo es presentar una revisión sobre todos los trabajos que han contribuido a mejorar la eficacia de este procedimiento, con la intención de darlo a conocer y promover estudios que indaguen en sus limitaciones (generalización y mantenimiento). Para ello, se presentan los aspectos generales del juego: organización de la clase, criterios para el refuerzo, dónde y cuánto tiempo dura el juego, tipo de refuerzos recibidos, comportamientos disruptivos a tratar; $y$ las diferentes aplicaciones que se han realizado del mismo. A modo de conclusión, parece que este

Correspondencia: Dra. M" del Rosario Ruiz Olivares. Departamento de Psicologia. Facultad de Ciencias de la Educación. C/ San Alberto Magno, s/n., 14071-CÓRDOBA. (SPAIN) TLF: 957-218900. FAX: 957-212513.- E-MAIL: m92rorum @uco.es. 
procedimiento se presenta muy eficaz para reducir la frecuencia de comportamientos disruptivos en contextos educativos.

Palabras clave: JUEGO DEL BUEN COMPORTAMIENTO, MODIFICACIÓN DE CONDUCTA GRUPAL, INTERVENCIÓN EN EL CONTEXTO ESCOLAR, COMPORTAMIENTOS DISRUPTIVOS.

\section{SUMMARY}

The Good Behavior Game (GBG), has as main objective to reduce the frequency of disruptive behavior in the class like: shauting, fighting, interrupting, etc. These behaviors are characterized by being functional in certain contexts like the playground or the free-game among partners and disfunctional in instructional-academic settings. The main aim of this article is to make a review about the published works that have contributed to implement its efficacy, and to promote some pieces of work that deals with their limits (generalization and management). The general aspects of the game are presented, class organization, support criteria where and how long the game will last, kind of support or reinforcements received, behaviour disruptive to deal with and the different applications that the Good Behavior Game. To conclude, it seems that this procedure is presented very efficiently in order to reduce the frequency of disruptive behaviour in educational contexts.

Key wOrds: GOOD BEHAVIOR GAME, MODIFICATION OF GROUP BEHAVIOR, SCHOOL CONTEXT INTERVENTION, DISRUPTIVE BEHAVIOR.

En la actualidad es frecuente escuchar a maestros y profesores, educadores en general, lamentarse acerca de las dificultades que suponen, en el proceso de enseñanza-aprendizaje, comportamientos como gritar, pelearse, falta de atención, insultos, etc, . ya que provocan continuas interrupciones y ralentizan dicho proceso. Esta realidad, tanto en Primaria como en Secundaria, perjudica a los alumnos, pues contribuye a su distracción, y a los profesores y maestros, creándose 
entre ellos una sensación de cansancio y falta de eficacia en su trabajo. Este tipo de comportamientos, a los que se ha hecho referencia (gritar, levantarse, pelearse, etc.) se denominan disruptivos y pueden ser definidos como aquellos que no resultan socialmente adaptativos porque dificultan o reducen tanto la probabilidad de integración del sujeto en el contexto educativo como la adquisición de repertorios que puedan ser reforzados. Estas conductas pueden ser clasificadas en agresivas, autoestimuladas, autolesivas e incompatibles (Luciano 1988). Concretamente, las conductas incompatibles se caracterizan por ser comportamientos que pueden resultar funcionales en ciertos contextos específicos (gritar, levantarse, saltar en el recreo o en alguna actividad de ocio), resultando disfuncionales en otros, por ejemplo en situaciones educativas, ya que impiden la emisión del comportamiento funcional requerido.

Teniendo en cuenta, que en la escuela se da una alta frecuencia de estas conductas disruptivas incompatibles y que los maestros se sienten limitados para intervenir recurriendo en última instancia a técnicas que resuelven de manera individual el problema, se hacen necesarios procedimientos que intervengan de forma específica en este contexto y para este tipo de comportamientos. En la literatura empírica, quedan recogidos varios procedimientos con el objetivo de disminuir aquellos comportamientos que se producen en exceso, como las técnicas de reforzamiento diferencial, el coste de respuesta, el tiempo fuera, la saciedad, la sobrecorrección y los procedimientos que utilizan la estimulación aversiva (Herruzo, Luciano y Pino, 2001). Normalmente, con la intervención individual se alcanza un nivel de satisfacción muy alto, sin embargo, puede resultar complejo manejar y mantener en el contexto escolar varias intervenciones orientadas a cada uno de los individuos, donde la mayoría de los alumnos presentan este tipo de comportamientos en una misma clase. En este sentido, las técnicas de contingencias orientadas hacia el grupo pueden ser tan efectivas y tan válidas como los procedimientos individuales para el cambio conductual (Tankersley, 1995). Además, resultan ideales para llevarlas a cabo en contextos como la escuela, pues le ahorran al maestro tiempo, facilita las interacciones sociales entre los miembros del grupo y ayuda en la construcción de comportamientos más apropiados (Gresham, 1983). 
Por contingencias orientadas hacia el grupo, se entiende aquel procedimiento en el que una conducta (o conductas) previamente establecidas, suponen la consecución o pérdida de un refuerzo basado en el comportamiento de un individuo dentro del grupo (Cooper, Heron y Heward, 1987; Alberto y Troutman, 1995). Dentro de este tipo de procedimiento, se pueden distinguir tres tipos: por un lado, las Contingencias Dependientes, en el que los miembros del grupo reciben el refuerzo si un individuo del mismo cumple el criterio establecido; las Contingencias Independientes, donde los miembros de un grupo que cumplen el criterio reciben un refuerzo; y las Contingencias Interdependientes, donde todos los miembros del grupo reciben refuerzo, si todos los miembros del grupo, o si el desarrollo del grupo como un todo, cumplen los criterios. En este último caso, las contingencias se hacen efectivas simultáneamente para todos, y cada individuo depende de lo que el grupo haya realizado en su conjunto (Cooper, et al., 1987).

Un ejemplo efectivo de este tipo de procedimientos es el Juego del Buen Comportamiento (JBC) (Good Behavior Game, GBG), que a través de un diseño por pequeños grupos consigue disminuir en gran medida la frecuencia de conductas disruptivas incompatibles en diferentes contextos educativos. Es decir, la puesta en marcha de esta técnica le garantiza al maestro una disminución considerable en la frecuencia de comportamientos disruptivos, como los que se han descrito anteriormente, mejorando el clima de convivencia y el trabajo del aula. Se trata de dividir un gran grupo de niños o jóvenes en dos o más equipos, con un serie de reglas especificas (que serían las conductas disruptivas objeto de estudio), de forma que los equipos reciban una marca en su contra si un miembro no cumple uina de esas normas. Se establece un criterio mínimo de posibles marcas y son reforzados todos los equipos que cumplen y no sobrepasan dicho criterio (Sulzer-Azaroff y Mayer, 1983).

Los primeros autores en desarrollar esta técnica y comprobar su eficacia fueron Barrish, Saunders y Wolf (1969). Estos autores se plantearon por un lado, comprobar los efectos de una posible técnica que mejorara la conducta del aula basada en refuerzos naturales de la misma clase, $u$ otros a los que el maestro le diera importancia; y por otro lado, desarrollar un procedimiento para reducir la conducta disruptiva en el aula a través de una competición a modo de juego con 
la idea de conseguir privilegios. Este primer intento por llevar a cabo este procedimiento fue todo un éxito y provocó que otros autores se interesaran por la técnica, de manera que se realizaran otros trabajos para demostrar su efectividad introduciendo ciertas modificaciones. Así, se pueden encontrar estudios centrados en la eficacia de los componentes del juego (Medland y Stachnik, 1972; Harris y Sherman, 1973); en la aplicación con población de alto riesgo (Grandy, Madsen y De Mersseman, 1973); su desarrollo en contextos diferentes a la escuela (Fishbein y Wasik, 1981); la puesta en marcha del procedimiento con adolescentes (Phillips y Christie, 1986; Salend, Reynolds y Coyle, 1989); con niños pequeños de preescolar y primero de primaria (Swiezy, Matson y Box, 1992; Doland, Kellam, Brown, Wethamer-Larsson, Rebok, Mayer, Landoff, Turkkan, Ford y Wheeler, 1993; Kellam, Ling, Merisca, Brown y lalongo, 1998); la evaluación de su eficacia comparándola con otro tipo de técnicas preventivas similares (Kellam, Mayer, Rebok y Hawkins, 1998b); y por último, también ha habido autores que han dedicado su esfuerzo a evaluar la aceptación de los maestros del JBC y si la edad de los niños o la severidad de las conductas problema influía en dicha aceptación (Tingstrom, 1994).

Sin embargo, ante este alarde de utilidad es necesario hacer referencia también a ciertas limitaciones que presenta este procedimiento como es la generalización de los resultados a otras conductas o a otros contextos no planificados, y el mantenimiento de los mismos en el tiempo. Además, cabe la duda si exclusivamente con el juego y su dinámica el niño adquiere un patrón conductual de autocontrol (Sulzer-Azaroff y Mayer, 1983). Por otro lado, Embry (2002) señala algunos problemas que se pueden presentar a la hora de aplicar el procedimiento, como es la falta de tiempo de los profesores/ maestros, las reticencias previas a la efectividad de la técnica, o la creencia de que los niños no deben ser reforzados por el comportamiento bien hecho.

\section{ASPECTOS GENERALES DEL JUEGo DEL BUEN COMPORTAMIENTO}

El Juego del Buen Comportamiento es una estrategia para mejorar el clima del aula, diseñada para aminorar las conductas disruptivas y 
prevenir en un futuro comportamientos más perjudiciales para la persona, cuya eficacia ha sido ampliamente demostrada desde el primer trabajo realizado por Barrish, et al. (1969), hasta nuestros días. Básicamente, dicho proceso consiste en dividir a los alumnos de una clase en dos o tres equipos, donde las reglas del mismo son aquellas conductas disruptivas objeto de estudio. Se trata de realizar una serie de sesiones de juego de corta duración, al final de las cuales y en función del comportamiento realizado, los niños recibirán una serie de contingencias. Durante el mismo, los niños reciben feedback en la pizarra sobre su comportamiento y el de sus compañeros (Barrish, et al., 1969).

Uno de los aspectos más destacado del procedimiento es que todos los equipos pueden ganar, si entre todos cumplen el criterio señalado del número de conductas permitido para cada sesión o juego. Es decir, que para ganar el juego no pueden superar un número concreto de comportamientos previamente establecido. Estos autores fueron los primeros en llevar a cabo esta técnica con estudiantes de cuarto curso de primaria, con los objetivos de reducir las conductas disruptivas más frecuentes en el aula y comprobar los efectos de esta técnica. En este caso, el procedimiento se llevó a cabo durante la hora de matemáticas y lengua, la clase se dividió en dos equipos y las reglas del juego fueron: no levantarse de su sitio sin permiso y no hablar sin permiso del profesor. Algunos de los refuerzos utilizados fueron llevar puestas tarjetas de victoria, poner una estrella en un panel especifico para tal efecto o tener treinta minutos de tiempo libre al final de la mañana. En cuanto al criterio de marcas para ganar que no debían superar los equipos en un principio, se comenzó con un máximo de diez marcas y finalmente, se acabó la intervención con un máximo de cinco.

A partir de este trabajo, el interés por desarrollar el procedimiento se acrecienta, tanto para comprobar la eficacia de los distintos componentes, como para introducir pequeñas modificaciones al mismo. No obstante, la mayoría de las replicaciones han sido consistentes con el formato original y el procedimiento desarrollado por estos primeros autores, de forma que, los componentes esenciales quedan de la siguiente manera (Tankersley, 1995):

- Las conductas objeto de estudio deben estar bien definidas y determinar cuándo estas conductas son más problemáticas en el aula. 
- Es conveniente establecer el criterio para ganar y los refuerzos que se van a establecer.

- Se les debe enseñar a los alumnos las reglas del juego.

- Debe quedar claro que pueden ganar todos los equipos que se formen.

- La clase se divide por equipos y los nombres de los mismos se colocan en un panel visible para todos, o en la pizarra.

- Si algún estudiante rompe una regla durante el juego, el profesor coloca una marca al lado del nombre del equipo al que pertenece dicho alumno.

- Cuando acaba el juego gana el equipo o los equipos que tengan el número de marcas criterio establecido o menos.

- Los miembros del equipo ganador obtendrán refuerzos no sólo por cada juego, sino que también podrán recibir refuerzos diarios y semanales.

Con respecto a los elementos, Harris y Sherman (1973) estudiaron sistemáticamente la mayoría de ellos, manipulando los refuerzos, el criterio máximo de errores, el feed-back que se establecía en la pizarra y la formación de los equipos. Encontraron que para que el juego fuera más efectivo, los profesores o la persona que desarrollara la técnica debía: establecer el criterio de conductas para ganar el juego; dividir la clase en equipos; adjudicar puntos a cada uno; y suministrar refuerzos a todos los miembros de los equipos ganadores.

A pesar de que la mayoría de los investigadores han seguido este procedimiento, los estudios han variado en la forma que se han organizado las clases, el criterio para el refuerzo, cuándo y cuánto tiempo debía durar el juego, el tipo de refuerzos y el método de aplicación a los ganadores, etc.

1.- En función de la organización de la clase: en este caso, muchos de los trabajos dividen a los alumnos en dos equipos atendiendo a la ubicación ya establecida en el aula, o a grupos de trabajo ya construidos (Barrish, et al., 1969; Medland y Stachnik, 1972; Harris y Sherman, 1973; Boston y Geiger, 1976; Hegerle, Kesecker y Couch, 1979; Fishbein y Wasik, 1981; Saigh y Umar, 1983). Sin embargo, los trabajos de investigación más recientes han optado por un modelo que divide la clase en tres equipos, siendo lo más heterogéneos posible (Salend, et al., 1989; Doland, et al., 1993; Kellam, et al., 1998b; Kellam, 
et al., 1998a: Patrick, Ward y Grouch, 1998; Babyak, Luze y Kamps, 2000; Van Lier, Muthen, Van der Sar y Crijnen, 2004). De estos últimos, cabe destacar el estudio llevado a cabo por Doland, et al. (1993), los cuales a pesar de formar equipos, individualizaron de alguna manera el proceso, formando grupos en función de las conductas disruptivas objeto de estudio que a nivel individual les caracterizaban. También destaca el trabajo realizado por Swiezy, et al. (1992) llevado a cabo con niños pequeños y donde la formación de los equipos quedaba reducida a una formación de parejas. A pesar de que la efectividad del juego con o sin equipos ha sido investigada por Harris y Sherman (1973), los cuales comprobaron que la técnica funcionaba mejor con la formación de grupos, también se han llevado a cabo estudios donde los alumnos eran considerados de manera individual, siguiendo el mismo procedimiento que se ha descrito hasta ahora (Grandy, et al., 1973; Webster, 1989).

Otro aspecto a tener en cuenta en la formación de los equipos es la reacción de cada uno de los estudiantes, ya que algunos alumnos intentan sabotear el esfuerzo realizado por sus compañeros. Ante esta problemática, se aportan diferentes alternativas: Barrish, et al. (1969) decidieron hacer un nuevo equipo con el único individuo que daba problemas, donde las contingencias pasaron de ser grupales a ser individuales. Por otro lado, Medland y Stachnik (1972) le dieron la oportunidad al propio equipo de votar para ver si se le permitía seguir participando o no en el juego durante un día completo al alumno que daba problemas. Y Harris y Sherman (1973) separaron los equipos de estudiantes que podían ser excesivamente reforzados por su conducta negativa del resto de compañeros, formando un solo equipo con ellos.

2.- En función de los criterios para el refuerzo: Cuando Barrish y sus colegas aplicaron por primera vez el JBC, establecieron como criterio máximo cinco marcas (Medland y Stachnik, 1972; Harris y Sherman, 1973; Boston y Geiger, 1976; Hegerle, et al., 1979; Saigh y Umar, 1983), aunque otros estudios más recientes lo bajaron hasta cuatro y tres marcas (Fishbein y Wasik, 1981; Doland, et al., 1993; Kellam, et al., 1998a; Kellam, et al., 1998b). Generalmente, los investigadores no han racionalizado el por qué de una cantidad o de otra, Harris y Sherman (1973) evaluaron los efectos de incrementar o 
aminorar el número de marcas necesarias. Estos autores sistemáticamente introdujeron el juego con un criterio inicial de ocho y lo cambiaron a cuatro. Los resultados mostraron que los estudiantes se comprometian con tantas conductas disruptivas como el criterio les permitía. Así, cuando el criterio era de cuatro, hubo un $50 \%$ menos de ocurrencia de las conductas objeto de estudio que cuando el criterio era de ocho.

3.- En función de dónde y cuánto tiempo se debe jugar. Normalmente, el juego se ha llevado a cabo en el contexto escolar, es decir, durante las horas lectivas (Babyak, et al., 2000; Barrish, et al., 1969; Boston y Geiger, 1976; Doland, et al., 1993; Grandy, et al., 1973; Hams y Sherman, 1973; Hegerle, et al., 1979; Johnson, Turner y Konarski, 1978; Kellam, et al., 1998a; Kellam, et al., 1998b; Medland y Stachnik, 1972; Phillips y Christie, 1986; Salend, et al., 1989; Swiezy, et al., 1992; Tingstrom, 1994; Saigh y Umar, 1983; Van Lier, et al., 2004; Wamer, Miller y Cohen, 1977; Webster, 1989). Sin embargo, destacan algunos trabajos que se han desarrollado en contextos diferentes como el elaborado por Fishbein y Wasik (1981) en una biblioteca, o el llevado a cabo por Patrick, et al. (1998) en una actividad deportiva como el voleibol. En cuanto al tiempo que debe durar una sesión de juego, existen casi tantas variaciones como estudios realizados. En este sentido, es conveniente señalar la importancia que adquiere el comenzar con un espacio de tiempo corto e ir aumentándolo hasta alcanzar la duración de las diferentes actividades cotidianas del contexto en el que se interviene. En esta línea, destaca el trabajo de Doland, et al. (1993) en el que sistemáticamente se fue incrementando el tiempo que los estudiantes jugaban al juego. Cuando el estudio comenzó, los alumnos jugaban durante diez minutos, tres veces a la semana y cada tres semanas la duración se incrementaba en diez minutos, hasta que los alumnos estuvieron jugando un máximo de tres horas diarias. El inconveniente que se desprende de esta dinámica es que el criterio en cuanto al número de conductas permitido no se vio incrementado proporcionalmente con el tiempo establecido. Es decir, que a los alumnos se les éxigía el mismo número de marcas, aunque el tiempo se viera ampliado, lo que suponía un gran esfuerzo para ellos.

4.- En función de los refuerzos que reciben los equipos ganadores: En este sentido, ocurre lo mismo que en criterios anteriores, ya que 
existe cierta variabilidad, aunque se persigan los mismos objetivos y se obtengan resultados similares. Así, desde refuerzos tangibles, hasta la realización de actividades lúdicas, o incluso, el aumento del tiempo libre, oscilan el tipo de refuerzo más utilizado en este tipo de trabajos. De nuevo, se hace especial atención al trabajo realizado por Harris y Sherman (1973), en el que investigaron la influencia de las consecuencias para los equipos ganadores en la efectividad de la técnica. En este sentido, cuando probaron a eliminar el refuerzo, la efectividad del mismo se vio considerablemente reducida. También es conveniente señalar que en adición al refuerzo diario, la mayoría de los investigadores han incorporado también refuerzos semanales (Doland, et al., 1993).

5.- En función de los comportamientos: Los resultados, en este sentido, han mostrado efectos muy positivos sobre las conductas observadas en el aula y consideradas como disruptivas, pero han sido menos consistentes en conductas académicas. Teniendo en cuerita los comportamientos sociales observados en el aula, se puede decir que las más comunes han sido las del tipo hablar sin permiso, hacer ruido excesivo, levantarse del pupitre sin permiso, insultar a los compañeros, etc., donde los resultados de la técnica han sido muy relevantes. Por otro lado, también es interesante señalar algunos trabajos en los que la conducta negativa ha sido ignorada para centrar el refuerzo exclusivamente en la conducta bien hecha (Fishbein y Wasik, 1981; Swiezy, et al., 1992; Webster, 1989). Dentro de las conductas sociales objeto de estudio de esta herramienta, hay que señalar el interés que se ha mostrado por indagar en cómo esta técnica influye o no en el desarrollo académico de los alumnos. Algunos investigadores no sólo han evaluado los efectos del JBC en comportamientos sociales, sino también en el fomento de conductas académicas. Los resultados presentan cierta variabilidad y no hay evidencias claras de que exista una influencia directa (Doland, et al., 1993). Sin embargo, si parecen darse ciertos efectos positivos secundarios en dichas habilidades académicas que se llevan a cabo en el aula (Harris y Sherman, 1973), pues parece fácil pensar que al producirse una mejora considerable en el clima del aula, se vea consecuentemente beneficiado el proceso de enseñanza-aprendizaje que se desarrolla en la misma. 


\section{APLICACIONES DE LOS PROCEDIMIENTOS DEL JBC}

Actualmente, se está en disposición de afirmar que esta técnica es una de las más efectivas a la hora de intervenir en el cambio conductual de comportamientos disruptivos en un grupo, en cualquier contexto (escuela, biblioteca, etc.) y con cualquier tipo de edad (preescolar, primaria o secundaria). Su fácil aplicación y su bajo coste económico hacen de este procedimiento una técnica muy necesaria y útil en contextos como la escuela.

Destacan algunos trabajos centrados en demostrar la eficacia de los diferentes componentes del juego, realizando un análisis sistemático de los mismos (reglas, feedback, grupos, etc.). Medland y Stachnik (1972) realizaron un trabajo con 28 niños de $5^{2}$ de Primaria, fragmentando la presentación de las reglas del juego (no salir de su sitio sin permiso del profesor, no hablar sin permiso del profesor y no interrumpir la atención o las actividades de otros compañeros). Es decir, en una primera fase de intervención se presentaron las reglas sin más; en una segunda fase, las reglas se dividieron en tres categorias y se dieron ejemplos de cada una; y por último, se mantuvo la referencia a las reglas pero sin juego. En cuanto a los resultados, parece que el hecho de fragmentar las reglas y dar ejemplo de las mismas, favorece la reducción de la frecuencia de las conductas objeto de estudio.

Por otro lado, Harris y Sherman (1972) se propusieron comprobar qué componentes del juego eran responsables del control de las conductas disruptivas. En este caso, llevaron a cabo el procedimiento normal en dos aulas de primaria, una de quinto y otra de sexto. En esta última fue donde se introdujeron ciertas modificaciones, fragmentando diferentes componentes del procedimiento, consiguiendo demostrar que las contingencias, el número de errores criterio para ganar los juegos y la división de los estudiantes por equipos tenian una gran importancia a la hora de reducir determinadas conductas.

También destaca el trabajo de Grandy, et al. (1973) que aplicaron el JBC a una población con alto riesgo de presentar un cuadro de fracaso escolar. En este sentido, desarrollaron el juego con alumnos que estaban cerca de presentar fracaso escolar, sobre todo en la clase 
de lengua (inglés), donde el clima de trabajo era insostenible. Por lo tanto, se decidió intervenir específicamente en dicha materia, aunque se tomaron datos también de la clase de aritmética que iba en el horario inmediatamente después. En este caso, las reglas del juego fueron formuladas de forma positiva: a) Levantar la mano para llamar la atención del maestro; b) Hablar sólo cuando se tiene permiso del profesor; c) Sólo se permite un alumno en la mesa del profesor; d) Permanecer cada uno en su sitio a menos que se tenga el permiso del profesor para levantarse. Los resultados mostraron un descenso del número de conductas recogido en la línea base en comparación con los datos de la fase de intervención.

A pesar de que la mayoría de los trabajos se han realizado en la escuela, cabe señalar algunos llevados a cabo en contextos diferentes a la misma, como el realizado por Fishbein y Wasik (1981) cuyo principal objetivo era llevar a cabo el JBC para conseguir disminuir la frecuencia de ciertas conductas disruptivas localizadas en una actividad extraescolar como era la biblioteca. Se intervino con 25 alumnos de $4^{2}$ de Primaria y las reglas fueron consensuadas con los usuarios y se formularon de forma positiva: a) Si hablas, hazlo suavemente; b) Elige un libro o mira los materiales durante el período de actividad en la biblioteca; c) Cuando andes, sé muy cuidadoso para no chocar con nadie; d) Trata al compañero con respeto siempre siendo cuidadoso de no empujarlo o herirlo. De nuevo los resultados confirmaron la eficacia de la técnica, a partir de los cuales se pudo ver un incremento motivacional de los estudiantes a comportarse de una forma más positiva. Parece que disminuyó el resentimiento entre los mismos y hubo un incremento de atención hacia el profesor con conductas positivas. Una de las conclusiones más interesantes que se vislumbra en este trabajo, es la posibilidad de aplicar este método en aquellas actividades semanales que se llevan a cabo fuera del horario escolar y que no cuentan con la constancia diaria de horario que tiene la escuela, pero que igualmente precisan de intervenciones similares a esta.

Por otro lado, destaca el trabajo desarrollado por Patrick, et al. (1998) en el que se pretendia aplicar la técnica del JBC sólo durante las horas que se impartía la materia de Educación Física, concretamente en la actividad de voleibol. Los objetivos planteados eran evaluar los efectos 
de la intervención sobre las habilidades sociales, y determinar si el número de pases aumentaba con la intervención realizada en dichas habilidades sociales. Los participantes fueron los alumnos de tres clases de primaria $\left(4^{2}, 5^{2}\right.$ y $\left.6^{2}\right)$ y fueron divididos en equipos de cinco o seis estudiantes. Durante las veinte lecciones que duró la intervención, cada una de las sesiones contó con diez minutos de JBC. Se observaron tres clases de conductas: el número de comportamientos sociales apropiados, el número de comportamientos sociales inapropiados, y el número de pases de antebrazo y saques correctos que se producían. Las conductas sociales positivas se dividieron a su vez en tres categorias: actos físicos, comportamientos verbales y comportamiento gestual de los alumnos. Con respecto a los resultados del estudio, se puede decir que en general, las conductas sociales inapropiadas se redujeron y se incrementaron los comportamientos sociales apropiados. Sin embargo, en lo que respecta a las habilidades técnicas del deporte, esta herramienta no pareció ejercer ningún tipo de influencia positiva. Esto puede deberse fundamentalmente a dos razones, primero, porque la respuesta social y motora no tienen por qué relacionarse entre sí, y segundo, porque, quizás, las condiciones de la línea base no fueron tan inapropiadas como para apreciar diferencias.

Otros trabajos han centrado sus esfuerzos en comparar el JBC con otro tipo de técnicas similares. En este sentido, destaca el trabajo realizado por Warner, et al. (1977), los cuales pretendian comparar el JBC con otro procedimiento centrado en promover la atención hacia el profesor. Cuatro aulas con 25 niños cada una y su respectivo tutor fueron los sujetos implicados en dicha intervención. En este caso, se vieron reducidas las conductas problema con ambos procedimientos, sin embargo al comparar ambas técnicas, parece que el JBC redujo de una forma más efectiva dichos comportamientos. Una de las explicaciones que se aporta ante este resultado es la facilidad de aplicación del mismo.

Dentro de esta misma línea, hay que mencionar el trabajo realizado por Tingstrom (1994), que pretendla evaluar la aceptación por parte de los maestros del JBC como un tipo interdependiente de contingencia de grupo y además, comprobar si la edad o la severidad de la conducta problema interfería de alguna forma en dicha aceptación por parte de 
los maestros. Concretamente, el JBC fue comparado con otro tipo de métodos: uno, denominado como Good Behavior Game Plus Merit y otro, basado en el coste de respuesta. Finalmente, el JBC fue igualmente aceptado por los maestros que el resto de métodos, caracterizados por aplicarse de una forma más individual. Por otro lado, en cuanto a la influencia de la edad y de la severidad de la conducta problema en la aceptación de uno u otro método, parece que fue nula.

Por último, Kellam et al. (1998a) también se marcaron el objetivo de comparar el JBC con otro método preventivo denominado Mastery Learning (ML), más interesado en el aprendizaje de contenidos académicos que en el cambio conductual. En este caso, la intervención duró dos años, a lo largo de los cuales se seleccionaron tres colegios al azar: en uno de ellos, se llevó a cabo la técnica del ML, en otro el JBC y finalmente, el tercero sirvió como colegio control externo. Las conductas objeto de modificación fueron: interrupción verbal, interrupción física, levantarse sin permiso y no obedecer. Ambas técnicas parece que contribuyeron a la disminución de la frecuencia de las conductas, pero el JBC lo hizo de una forma más consistente que en el caso del ML. En este último procedimiento, el cambio conductual resultó ser más un reflejo de la mejora que se logró a nivel académico, que de la propia técnica en sí. Además, en este caso se observó un cambio de las conductas disruptivas en los niños, pero no en las niñas.

El trabajo llevado a cabo por Saigh y Umar (1983) es digno de destacar también, ya que aplica esta técnica en una cultura diferente a la descrita en la mayoría de los estudios. Dichos autores se plantearon llevar a cabo el juego en un colegio público rural localizado en la República Democrática de Sudán. La intervención fue realizada en una clase de segundo de Primaria con 20 alumnos, de los cuales el $80 \%$ de sus padres eran analfabetos y el $85 \%$ de los estudiantes se veían obligados a trabajar con sus familias en fábricas. Las conductas disruptivas localizadas en la dinámica del aula fueron muy similares a las que hasta ahora se han estado describiendo: hablar o interrumpir verbalmente, agredir o interrumpir físicamente y levantarse del asiento sin permiso. Los resultados confirmaron que el JBC tiene un efecto positivo en la modificación de estas conductas en los alumnos. Por lo que se puede afirmar, que la cultura no parece influir en el desarrollo del método ni en la obtención de resultados positivos. 
Es interesante hacer mención a los trabajos que se han llevado a cabo con adolescentes, y concretamente, con aquellos que presentan ciertas dificultades. Así, destaca un primer trabajo elaborado por Phillips y Christie (1986), los cuales desarrollaron el método en un aula que respondía a lo que en el sistema educativo español se conoce como «Diversificación Curricular». Concretamente, en este estudio, se trataba de 19 chicos y 9 chicas con edades comprendidas entre los 12 y 13 años, con la costumbre de realizar conductas molestas que favorecían la creación de un clima de trabajo poco adecuado (hacer ruidos, saltar en sus asientos, golpear a otros compañeros, etc.). En ese caso, las reglas también fueron consensuadas entre la maestra y los alumnos: a) Cuando el profesor esté hablando, nosotros atendemos, b) Pedimos permiso para hablar levantando la mano, c) Intentamos no interrumpir a otros compañeros que estén trabajando, d) Desarrollamos nuestro propio trabajo tranquilamente, y e) Intentamos poner atención a nuestro trabajo y procuramos no distraernos. En general, se puede decir que el clima de clase mejoró considerablemente y como consecuencia parece que el rendimiento académico de los alumnos también.

En un segundo trabajo, realizado por Salend, et al. (1989) se pretendía llevar a cabo el JBC con estudiantes de entre 15 y 17 años emocionalmente perturbados. En este caso, los alumnos fueron divididos en grupos en función de la conducta disruptiva que más frecuentemente realizaban. El estudio se llevó a cabo con tres clases, en cada una de las cuales se formaron dos o tres equipos teniendo en cuenta esa conducta más frecuente. Los comportamientos disruptivos considerados de manera individual se redujeron notablemente con la aplicación de esta técnica.

Este procedimiento también se ha llevado a cabo con alumnos pequeños de educación especial, destacando el trabajo realizado por Webster (1989) con nueve chicos de edades entre los 9 y 10 años, considerados inmaduros con respecto a su edad y con dificultades sociales y académicas. En este caso, las tres reglas que marcaban el juego eran: a) Hablar suavemente a la persona que tenemos cerca, b) Permanecer en nuestro asiento y no levantarnos sin permiso del profesor, y c) No pelearse con el resto de compañeros. Se introdujo en la dinámica de la técnica el coste de respuesta, pues cada vez que 
algún miembro del equipo no cumplía las reglas, al grupo clase le restaban un punto positivo. La mejora en los comportamientos de los niños fue significativa y muy positiva al crear un clima de aula más adecuado a las necesidades del proceso de enseñanza-aprendizaje.

Swiezy, et al. (1992) han sido los primeros en desarrollar este procedimiento con niños de preescolar de edades comprendidas entre los 4 y los 5 años que presentaban problemas interpersonales y de conducta, aspectos con una influencia negativa en su habilidad para cooperar. En este caso, al ser niños muy pequeños se requería un mayor esfuerzo por parte del maestro para llevar a cabo la intervención. En cuanto a las conductas objeto de la intervención, se pueden resumir en obediencia y no obediencia, siendo reforzado el cumplimiento de una instrucción dada por el maestro a través de un oso de peluche, e ignorado el no cumplimiento de la misma. Parece que las conductas problema disminuyeron notablemente y aumentó el rendimiento académico y la atención al maestro.

Doland, et al. (1993) también trabajan con población infantil y en este caso, se seleccionaron al azar cuatro colegios, de los que fueron elegidas las clases de primero de Primaria, con un total de 182 estudiantes de 6 a 7 años. Como en trabajos anteriores, las conductas objeto de la intervención fueron la interrupción verbal, física, levantarse del pupitre sin permiso y no obedecer. En este caso, las conductas disruptivas fueron evaluadas por el maestro y los propios compañeros al principio y al final de la intervención. Los resultados fueron de nuevo positivos, mostrando una disminución considerable de las mismas.

Por último, Kellam, et al. (1998b) también aplicó el método del JBC con niños de primer curso para prevenir la conducta agresiva de 238 niños. El trabajo utilizó en total 19 colegios de los cuales, 168 alumnos fueron considerados controles internos, 238 niños objeto de la intervención y 308 alumnos considerados como controles externos. El tratamiento preventivo de las conductas agresivas en la escuela parecen quedar paliadas con la puesta en marcha de esta técnica. De estos últimos trabajos, cabe destacar la inclusión tanto en la dinámica de la intervención como en el diseño de la misma, de grupos controles de carácter interno (dentro del mismo colegio) y de carácter externo (localizados en otra serie de colegios). 
Después de esta revisión bibliográfica, parece inevitable confiar en la eficacia de este procedimiento, independientemente del contexto, de la población, de la edad, de las características especiales de los alumnos, etc. Sin embargo, hay aspectos como la generalización espontánea a otros contextos o conductas, y el mantenimiento de los resultados que son, aún, tareas pendientes de esta línea de investigación.

\section{ASPECTOS DE GENERALIZACIÓN Y MANTENIMIENTO DEL JBC}

En la mayoría de los trabajos cuyo objetivo es desarrollar y ampliar, de alguna manera, esta técnica, apenas se encuentran referencias a los procedimientos utilizados para la generalización o el mantenimiento del aprendizaje adquirido. Ambos aspectos son considerados vitales, si se tiene en cuenta que una de las ventajas de este método es el bajo coste económico de la misma. De esta manera, cabe pensar que un procedimiento será aún más estable, si lo que enseña se mantiene en el tiempo y si además, la persona es capaz de generalizar dicho procedimiento de forma espontánea (aportándole las variables necesarias en la intervención) y natural a otras situaciones y comportamientos nuevos.

Con respecto a la generalización, destacan dos trabajos que hacen referencia a la misma, pero sin aplicar ningún procedimiento concreto para conseguir tal efecto. Por un lado, en el estudio desarrollado por Grandy, et al. (1973) se tiene en cuenta la generalización del patrón de conducta aprendido hacia otra materia pero en el mismo contexto escolar. Así, se aplica la intervención en la clase de lengua con la idea de que los resultados se generalicen a la clase posterior de aritmética de una forma casi espontánea. Para constatar dicha generalización, desde el principio se recogen los datos de una línea base múltiple, donde en el análisis se percibe cómo cada vez que se interviene en la clase de lengua las conductas disruptivas disminuyen en la clase de aritmética, y cómo cuando desaparećan las contingencias en la clase de lengua, la frecuencia de las conductas disruptivas de la clase de aritmética volvía a su estado habitual. Este es el indicio al que ellos apuntan sobre la generalización de los resultados.

Por otro lado, también está el trabajo de Swiezy, et al. (1992) donde de nuevo se hace referencia al aspecto de la generalización de los 
resultados del JBC a otros contextos (contexto de intervención, contexto normal). Sin embargo, la generalización no se dio en los diferentes contextos, sino que se produjo con respecto a las dos terapeutas implicadas en la intervención. No obstante, no hay un procedimiento claro y elaborado a partir del cual se pueda dilucidar los entresijos y las variables necesarias para tal generalización.

A pesar de la necesidad de conocer qué elementos promueven la generalización de este método, y el intento por parte de algunos de estos trabajos por averiguar dichos elementos, lo cierto es que no hay datos que apoyen un procedimiento de generalización de la conducta aprendida.

Por otro lado, en lo que respecta al mantenimiento de los resultados a lo largo del tiempo, ocurre lo mismo, en muchos de estos estudios se hace referencia a esta necesidad; sin embargo, en este caso, ni siquiera existen trabajos que tengan en cuenta este aspecto de forma explícita y aplicada.

\section{CONCLUSIONES}

La revisión bibliográfica realizada en torno a esta técnica de contingencia con grupos, como es el Juego del Buen Comportamiento, muestra la eficacia de la misma, pues la mayoría de los trabajos cumplen el objetivo de reducir la frecuencia de comportamientos disruptivos de tipo incompatible en niños y adolescentes en contextos educativos.

A lo largo de los diferentes trabajos, se ha puesto de manifiesto que la aplicación de este tipo de procedimientos, es muy positivo cuando determinados comportamientos, como gritar, interrumpir, levantarse constantemente del pupitre, pelearse, etc. interrumpen el proceso de enseñanza-aprendizaje. Hoy por hoy, la escuela presenta una realidad compleja, donde maestros y profesores se ven desbordados a la hora de llevar a cabo su trabajo, ya que estos comportamientos disruptivos en niños y jóvenes interrumpen constantemente la dinámica del mismo. Ante esta situación, los maestros precisan manejar procedimientos como el Juego del Buen Comportamiento, a partir del cual puedan establecerse unas normas de convivencia y bienestar para todos. 
La literatura empírica deja constancia de que este procedimiento puede ser una respuesta adecuada a dicha realidad escolar, ya que se caracteriza por su eficacia, la facilidad con la que se puede llevar a cabo, su bajo coste económico, la puesta en práctica por el propio maestro, la variedad de contextos en los que puede llevarse a cabo, las diferentes edades y realidades educativas con las que se puede aplicar, su versatilidad, flexibilidad, etc. Todos ellos son aspectos que, sin duda, la convierten en una técnica digna de ser estudiada con mayor profundidad. Además, su intervención con pequeños grupos dentro del gran grupo de la clase, no sólo consigue disminuir la frecuencia de comportamientos disruptivos, como los que se han enumerado a lo largo de este artículo, sino que, también puede llegar a fomentar comportamientos de solidaridad, compañerismo, ayuda, etc., aspectos que favorecen sensiblemente, el clima del aula y las relaciones interpersonales que en ella acontecen.

Es interesante también señalar como un aspecto positivo más la idea de que el JBC llegue a ser un instrumento fundamental en la prevención de aspectos tan preocupantes en la juventud actual, como el consumo de drogas, la agresividad, el fracaso escolar, etc. Hoy en día, la idea de la prevención desde una edad temprana parece la solución más viable (Embry; 2002).

Sin embargo, ante este alarde de utilidad, y como ya se ha hecho referencia, este importante procedimiento también presenta ciertas limitaciones, que no son, sino aspectos pendientes para ser objeto de estudio. En este caso, hay que hacer referencia a la generalización del patrón de conducta aprendido a otros comportamientos o a otros contextos diferentes al de la intervención (Grandy, et al., 1973; Swiezy, et al., 1992); y el mantenimiento de los resultados a lo largo del tiempo. Otros aspectos también interesantes, aún pendientes por explorar, son el uso de la técnica para la integración de alumnos con Necesidades Educativas Especiales; la mejora del rendimiento académico dentro de las diferentes materias del currículum escolar; o el desarrollo de habilidades sociales entre compañeros.

En resumen, se puede decir que el Juego del Buen Comportamiento, en el que los alumnos tienen que controlar su propia conducta y la de sus compañeros de equipo, es un procedimiento realmente efectivo para el cambio conductual de niños y jóvenes, sobre todo cuando se 
hace referencia a conductas disruptivas, como gritar, pelearse, levantarse sin permiso, etc. Un procedimiento de contingencia orientado hacia el grupo de forma interdependiente, puede llegar a ser muy beneficioso para el trabajo del maestro en la escuela, y puede ser una técnica realmente positiva, fácil y dinámica de llevar a cabo por él mismo. Por último, es necesario señalar, que no hay trabajos realizados con población española que confirmen dicha eficacia, por lo tanto sería muy interesante ampliar horizontes y desarrollar dicha línea de investigación más allá del mundo anglosajón.

\section{REFERENCIAS BIBLIOGRÁFICAS}

Albert, P.A. y Troutman, A.C. (1995). Applied Behavior Analysis for Teachers Englewood: Prentice-Hall.

Babyak, A., Luze, G. y Kamps, D. (2000). The Good Student Game: behavior management for diverse classroom. Intervention in School and Clinic, 35, 216-223.

Barrish, H., Saunders, M. y Wolf, M. (1969). Good Behavior Game: Effects of individual contingencies for group consequences on disruptive behaviour in a classroom. Joumal of Applied Behavior Analysis, 2, 119-124.

Boston, D. y Geiger, G. (1976). Good Behavior Game: a replication and systematic analysis with a second grade class. School Applications of Leaming Theory, 8(2), 18-27.

Cooper, J.O., Heron, T.E. y Heward, W.L. (1987). Aplied Behavior Analysis. Nueva York: MacMillan.

Doland, L., Kellam, S, Brown, C., Werthamer-Larsson, L., Rebok, G., Mayer, L., Laudolff, J., Turkkan, J., Ford, C. y Wheeler, L. (1993). The short-term impact of two classroom-based preventive interventions on aggressive and shy behaviours and poor achievement. Joumal of Applied Developmental Psychology, 14, 317-345.

Embry, D. (2000). The pax acts games solution: applying replicated research and current evaluation from the Good Behavior Game for achievement and prevention in schools. A special presentation to the national crime prevention council. Paradigms, 1, 1-20. (http: // www.paxis.org). 
Fishbein, J. y Wasik, B. (1981). Effect of the Good Behaviour Game on disruptive library behaviour. Joumal of Applied Behavior Analysis, 14, 89-93.

Grandy, G. Madsen, C. y De Mersseman, L. (1973). The effects of individual and interdependent contingencias on inapropiate classroom behavior. Psychology in the Schools, 10(4), 488-493.

Gresham, F.M. (1983). Social skills training with handicapped children: A review. Review of Educactional Research, 51, 139-176. Harris, W. y Sherman, J. (1973). Use and analysis of the "Good Behavior Game" to reduce disruptive classroom behaviour. Hegerle, D., Kesecker, M. y Couch, J. (1979). A behavior game for the reduction of inapropriate classroom behaviors. School Psychology Digest, 8(3), 339-343.

Herruzo, J. y Pino, M.J. (2001) Disminución de conductas disruptivas mediante un procedimiento de correspondencia decir-hacer. Acta Comportamentalia, 1(9), 145-162.

Johnson, M., Turner, P. y Konarski, E. (1978). The «Good Behavior Game": A systematic replication in two unruly transtional classrooms. Education and Treatment of Children, 1(3), 25-33.

Kellam, S., Ling, X., Merisca, R., Brown, C. y lalongo, N. (1998). The effect of the level of aggression in the first grade classroom on the course and malleability of aggressive behaviour into middle school. Development and Psychopathology, 10, 165-185.

Kellam, S., Mayer, L., Rebok, G y Hawkins, W. (1998). Effects of improving achievement on aggressive behaviour and of improving aggressive behaviour on achievement through two preventive interventions: an investigation of casual paths. En B. Dohrennend (Ed.) Adversity, stress and Psychopathology. Londres: Oxford University Press, XV.

Luciano, M.C.(1988). A systematic replication of response prevention to eliminate nocturnal thumbsucking. Child and Family Behavior Therapy, 10 (2/3): 69-75.

Medland, M. y Stachnik, J. (1972). Good-Behavior Game: A replication and systematic analysis. Journal of Applied Behavior Analysis, 5, 45-51.

Patrick, C., Ward, P. y Grouch, D. (1998). Effects of holding students accountable for social behaviors during volleyball games in 
elementary physical education. Journal of Teaching in Physical Education, 17, 143-156.

Phillips, D. y Christie, F. (1986). Behavior management in a secondary school classroom: playing the game. Maladjustment and Therapeutic Education, 4(1), 47-53.

Saigh, P. y Umar, A. (1983). The effects of a Good Behavior Game on the disruptive behavior of sudanese elementary school students. Journal of Applied Behavior Analysis, 16, 339-344.

Salend, S., Reynolds, C. y Coyle, E. (1989). Individualizing the Good Behavior Game across type and frequency of behaviour with emotionally disturbed adolescents. Behavior Modification, 13(1), 108-126.

Sulzer-Azaroff, B. y Mayer, G.R. (1985). Procedimientos del Análisis Conductual Aplicado con Niños y Jóvenes. México: Editorial Trillas. Swiezy, N., Matson, L. y Box, P. (1992). The Good Behavior Game: a token reinforcement system for preschoolers. Child and Family Behavior Therapy, 14(3), 21-32.

Tankersley, M. (1995). A group-oriented contingency management program. Preventing School Failure, 40(1), 1-7.

Tingstrom, D. (1994). The Good Behavior Game: an investigation of teachers' acceptance. Psychology in The Schools, 31, 57-65.

Van Lier, P., Muthen, B., Van der Sar, R. y Crijnen, A. (2004). Preventing disruptive behavior in elementary schoolchildren: impact of a universal classroom-based intervention. Journal of Consulting and Clinical Psychology, 72(3), 467-478.

Warner, S., Miller, F. y Cohen, M. (1977). Relative effectiveness of teacher attention and the "Good Behavior Game» in modifying disruptive classroom behaviour. Journal of Applied Behavior Analysis, 10, 737-738.

Webster, J. (1989). Applying behaviour management principles with limited resources: going it alone. Maladjustment and Therapeutic Education, 7(1), 30-38. 